\title{
Active Contour Based Medical Image Segmentation and Compression Using Biorthogonal Wavelet and Embedded Zerotree
}

\author{
R. Loganathan ${ }^{1 *}$ and Dr. Y. S. Kumaraswamy ${ }^{2}$ \\ 'Research Scholar, Sathyabama University, Chennai, Tamilnadu, logu73@yahoo.com \\ ${ }^{2}$ Sr. Prof. \& HOD, Dept. of MCA, DSCE, Bangalore, Karnataka, yskldswamy@yahoo.co.in
}

\begin{abstract}
This paper addresses medical image compression, as more and more medical images are digitized, economical and effective data compression technologies are needed to minimize the storage volume of medical database in hospitals. In this paper, the Region of Interest (ROI) - representing the diseased part - in a medical image is segmented using active contours. The ROI extracted are then compressed using lossless compression to maintain the integrity. A novel Biorthogonal wavelet and Embedded ZeroTree (EZW) is proposed for compression technique. Experimental results demonstrate that the proposed method significantly improves the Peak Signal to Noise Ratio (PSNR) for the medical image compression.
\end{abstract}

Keywords: Medical Images, Image Compression, Region of Interest, Active Contour, Biorthogonal Wavelets, Embedded ZeroTree (EZW).

\section{Introduction}

Modern radiology techniques give medical information to radiologists to diagnose diseases and to determine appropriate treatment. Such information should be acquired through medical imaging procedures. As more and more medical images are digitized, economical and effective data compression technologies are needed to minimize of digital image data mass volume in hospitals [1]. Compression schemes can be categorized into two categories: lossless and lossy compressions. Lossless image compression is achieved if original input image is recovered perfectly from compressed data while lossy image compression cannot regenerate original image data. Lossy image compression, can maintain most details of original image, useful for diagnosis. Precise image detail preservation is not strictly required as an image's degraded portion is usually often not visible to humans. But lossy image compression is not com- monly used in clinical practice/diagnosis because even with slight loss of data, it is possible for physicians/radiologists to miss critical diagnostic information that is needed for diagnosis of a patient [2].

Historically, medical image compression was investigated by researchers working in the image and video coding fields. Consequently, technological growth in this field is a by-product of progress in the more general field of natural image and video coding. There is no golden rule: different coding algorithms fit different types of images and scenarios best. Depending on imaging modalities and applications, some are better suited than others to fulfil certain targets in terms of compression factor or with respect to a desired functionality. A challenging requirement concerns the visually lossless mode.

Compression is not about storage costs alone. It is also about transmission time, imaging apparatus utilisation and the patient's convenience/comfort. Compression techniques

* Corresponding author:

Loganathan R (logu73@yahoo.com) 
by reducing file size and transmission time can thereby improve overall care. Image compression techniques take advantage of any occurring redundancy. There are different redundancy types and each compression methodology exploits one of these. The different redundancy types are spatial, temporal and spectral.

Present compression schemes have high compression rates when quality loss can be afforded. But physicians cannot afford deficiencies in image regions which are important, known as Regions of Interest (ROIs). An approach which brings high compression rates accompanied by good quality in ROIs is needed. A common idea is to preserve quality in diagnostically critical regions while allowing lossy encoding of other regions. The research's aim focuses on ROI coding to ensure use of multiple and arbitrarily shaped ROIs in images, with arbitrary weights describing the importance for each ROI including background (i.e. image regions not of ROI) so that latter regions can be represented by varying levels of quality [3].

Various compression methods for retaining maximum energy have been proposed in literature, improving the compression ratio. To overcome the limitations of lossy compression, ROI of the image is compressed using different techniques. In compression techniques using wavelet transforms, a low pass and a high pass filter are so chosen that it halves the frequency range between them. Compression algorithms include Shapiro's EZW (Embedded Zerotree Wavelet) [4], the SPIHT (Set partitioning in Hierarchical Trees) [5], and SPECK (Set Partitioning Embedded Block) [6]. These algorithms rely on embedded coding for an embedded binary flow, a progressive data transmission allowing image reconstruction through various compression ratios.

Tamilarasi et al. [7] proposed an approach, a wavelet based contourlet image compression algorithm that endeavours while preserving the diagnostic integrity, to decrease the transmission cost. Superior performance of contourlet transform is revealed in current reports on natural image compression. The essential part (ROI) in the diagnosis of medical images is divided from the other parts of the image by implementing neural network based fuzzy logic technique using a comparison of the test image with the training patterns and next, in order to improve the visual quality, the resultant image is optimized by applying contourlet transform. In order to obtain the compressed image the resultant image Huffman coding is applied. Jilani et al. [8] proposed a novel method for image processing that is a fuzzy optimization design based on neural networks.
Approximately 30\% more accuracy in retrieving image was obtained by the Embedded zero tree wavelet coding with fuzzy Back propagation artificial networks when compared to the existing EZW coding system.

Christophe et al. [9] focused on the optimization of a full wavelet compression system. Every step is studied and optimized in the compression algorithm. With the near-optimal decomposition, two effective compression methods based on zerotree coding (EZW and SPIHT) are adapted that reveals the best tree structure. With the adaptation of JPEG 2000, the performances are compared on six different areas presenting various statistical properties for hyperspectral images. Shingate et al. [10] proposed to compress an image into a bit stream with improving accuracy with EZW encoder based on progressive encoding. Using the EZW scheme coding of an image is performed along with few optimizations results in an image compressor that is amazingly efficient possessing the property of compressed data stream with any bit rate preferred. Using an EZW encoder, lossless compression is also possible but the results obtained are with only less spectacular. Hence, the proposed approach uses zero tree structure of wavelet coefficients in effective manner resulting in superior compression ratio and best PSNR and SNR. Babu et al. [11] proposed an approach using Partial EZW Algorithm for an enhanced image compression method. The difficulty of EZW which tends to lose its effectiveness of lower bit planes transmission is prevailed over by the proposed Partial EZW Algorithm. Additionally, the incorporation of wavelet transformation and region of interest coding to Partial EZW is included in this paper and therefore a best coder is obtained than the EZW and SPIHT Algorithm. The results are proved and validated. The proposed coder in terms of PSNR and CR reveals better performance for medical images.

Symmetric image border extension achieves better compression than periodic extension. The DWT require symmetric filters for using non-expansive, symmetric extensions, thus, orthogonal wavelets are not feasible for image compression as orthogonality and symmetry cannot be simultaneously possessed [12]. The capability of symmetric extension with biorthogonal wavelets achieves better performance for image compression applications.

In this paper, it is proposed to segment ROI using Active Contour. A novel Biorthogonal wavelet and Embedded ZeroTree (EZW) wavelet coding is proposed for compression technique. The rest of the paper is organized as follows: section 2 deals with related works, section 3 explains the 
techniques used in this paper, section 4 tabulate the results and section 5 concludes the paper.

\section{Materials and Methods}

\subsection{Active Contour}

From the introduction of snakes [13], active contours were applied to various problems, like image segmentation, feature extraction and image registration. The first active-contour methods were edge-based, using functionals which depended on image response to an edge filter $[14,15]$ such that the size of motion forces derived from functionals is small when edge strength is large. Segmentation with an edge-based active contour can be affected by edge detector problems.

In active contour systems, a contour is generally associated with minimal energy when a contour coincides with segmented object boundary. Widely used energy types rely on boundary curve smoothness and on image features. Image features refer to the edge strength, yielding edge-based active contours, or to the region's characteristics occupied by processed image objects, yielding region-based active contours. The segmentation process begins with an initial contour received automatically or through user interaction and is evolved toward object boundaries under forces derived from the energy, energy minimization being accomplished by gradient descent.

The energy types usually rely on boundary curve smoothness and on image features. The segmentation process begins with an automatic contour or through user interaction. This contour is evolved in the direction of object boundaries under forces derived from the energy and the energy minimization is obtained by gradient descent. The active-contour methods are mostly edge-based, using functionals which depends on image response to an edge filter [16].

The basic Active model is given by

$$
\begin{aligned}
F\left(c^{+}, c^{-}, C\right)= & \mu \cdot \text { Length }(C) \\
& +\lambda^{+} \int_{\text {inside }(c)} \mid u_{0}(x, y)-c^{+} \mathbf{I}^{2} d x d y \\
& +\lambda^{-} \int_{\text {outside }(C)}\left|u_{0}(x, y)-c^{-}\right|^{2} d x d y
\end{aligned}
$$

where, $\mathrm{c}+$ and c- are constant unknowns representing the average value of $\mathrm{u} 0$ inside and outside the curve, respectively. The parameters $\mu>0$ and $\lambda^{+}, \lambda^{-}>0$ are weights for the regularizing term and the fitting term, respectively. The above model can be rewritten as

$$
\begin{aligned}
F\left(c^{+}, c^{-}, \emptyset\right)= & \mu \cdot \text { Length }\{\varnothing=0\} \\
& +\lambda^{+} \int_{\varnothing<0}\left|U_{0}(x, y)-c^{+}\right|^{2} d x d y \\
& +\lambda^{-} \int_{\varnothing<0}\left|U_{0}(x, y)-c^{-}\right|^{2} d x d y
\end{aligned}
$$

for the level set formation where, $c=\{(x, y) \in \Omega: \emptyset(x, y)=0\}$

\subsection{Biorthogonal Wavelets}

A complete Biorthogonal wavelets family is built through variations of the three lifting stages:

\subsubsection{Split}

Other choices but Lazy wavelets are possible as initial split. A general alternative is Haar wavelet transform.

\subsubsection{Predict}

In the parlance of wavelets, prediction establishes the number of vanishing moments $(\mathrm{N})$ of a dual wavelet. It can also be stated that if the original signal is a polynomial of degree less than $\mathrm{N}$, all wavelet coefficients are zero.

\subsubsection{Update}

In wavelet terminology, update establishes vanishing moment's number (e N) of primary l wavelet. It means that the transform preserves first e $\mathrm{N}$ moments of $\mathrm{j}$ sequences.

Wavelets are a class of functions which satisfy specific mathematical requirements and represent data or various other functions. Using this superposition of functions to approximate other functions is not new. In the 1800s, Joseph Fourier discovered that any periodic function could be expressed as sum of sines and cosines; which today is Fourier analysis. Wavelets are extensions of Fourier analysis. Wavelet analysis aims to turn signal information into numbers-coefficients-which can be manipulated, stored, transmitted, analyzed, or reconstruct the original signal.

The Biorthogonal scaling function is given by [17]:

$$
\begin{gathered}
\phi(t)=2 \sum_{n=-\infty}^{\infty} h(n) \phi(2 t-n) \quad \text { dual } \tilde{\phi}(t)=2 \sum_{n=-\infty}^{\infty} \tilde{h}(n) \tilde{\phi}(2 t-n) \\
\langle\phi(t), \tilde{\phi}(t-k)\rangle=\delta(k) \quad\left\langle\phi\left(2^{-k} t\right), \tilde{\phi}\left(2^{-k} t-n\right)\right\rangle=2^{k} \delta(n)
\end{gathered}
$$

where, $h(n)$ and $\check{h}(n)$ - impulse response of FIR filters and

$\phi(t)$ and $\tilde{\phi}(t)$ - two sets of scaling functions which generate subspaces. 


\subsection{Embedded ZeroTree (EZW)}

Embedded ZeroTree wavelet coding is an effective, computationally inexpensive technique to compress images [18]. The principles of computation algorithm are (1) wavelet pyramid decomposition of image, (2) partial ordering of transform coefficients by highest bit plane of magnitude, with ordering information being encoded by a set partitioning algorithm reproduced at the decoder, (3) ordered bit plane transmission of refinement bits, and (4) exploitation of self-similarity of image wavelet pyramid decomposition across different scales.

An EZW encoder is specially designed for use with wavelet transforms. This is the reason why it includes 'wavelet' in its name. The encoder was basically designed to operate on images (2D-signals) but could also be used for other dimensional signals.

The EZW encoder is founded on progressive encoding to compress images into bit streams with improved accuracy. This means that when more bits are added to the stream, the decoded image would contain many more details, a characteristic similar to JPEG encoded images.

The EZW encoder is based on two observations:

1. Natural images have a low pass spectrum. When an image is wavelet transformed, the sub bands energy decreases as scale decreases (low scale means high resolution); hence wavelet coefficients will be smaller in the higher sub bands on average than in lower sub bands. This proves that progressive encoding is an automatic choice to compress wavelet transformed images, as higher sub bands only provide more details;

2. Large wavelet coefficients carry more importance than smaller wavelet coefficients

Embedded zeroTree wavelet algorithm (EZW) is a simple and effective image compression algorithm, with a characteristic that bits in a bit stream are generated in an order of importance, to yield a fully embedded code which in turn represents a sequence of binary decisions distinguishing an image from a "null" image. With an embedded coding algorithm, an encoder terminates encoding at any given time allowing a target rate/target distortion metric to be met on the dot. Also, with a bit stream, a decoder can cease decoding at any time in a bit stream but still produce the same exact image which would have been encoded at a bit rate matching a truncated bit stream. In addition to production of a fully embedded bit stream, EZW regularly provides competitive compression results with all compression algorithms on test images. What is surprising is that this is achieved by a technique requiring no training, no pre-stored tables or codebooks, and no prior knowledge of image source.

The major steps in generating zero-tree are:

1. Determining threshold $\mathrm{T}$ for iteration.

2. Scanning the image and comparing coefficient magnitude with respect to $\mathrm{T}$. Within the quad-tree in the sub bands, coefficients are roots of zero-trees.

3. Scanning through coefficients to generate a symbol representing the coefficient sign if its magnitude is greater than T. For zero-trees, a zero-tree symbol is put out at the root, to identify the whole quad-tree as zero. The scan order ensures that approximation signal is first scanned, followed by sub bands.

4. Coefficients exceeding the threshold in present iteration is set to zero as later iterations, need to only send the coefficient sign once; but successive iterations add bits to the coefficient en-coding leading to better and highly accurate value

5. Refining threshold by reducing it by half and iterating it through steps 2-4.

As Zero-tree represents visually less significant image regions, watermark bits can be placed at zero-tree coefficient without visual degradation of original image quality. As zero-tree and watermark are both scattered over the image, the image is thus made robust.

\section{Results and Discussion}

The proposed method was evaluated in MATLAB platform. Figure 1 shows the input and output for different medical images where the mask for the ROI between the important space and the non-important space is computed. Figure 2 shows the extracted ROI and Non ROI part of the image.

Figure 3 shows the Non ROI image after compression.

The bits per pixel obtained are tabulated as shown in Table 1 and is compared with SPITH.

Using EZW method, the medical image is segmented into ROI and non ROI, it is seen that the significantly improved Peak Signal to Noise Ratio (PSNR) is obtained. A higher value of PSNR means that the ratio of Signal to Noise is higher. The 'signal' refers to the original image, and the 'noise' is the error in reconstruction of the image. 

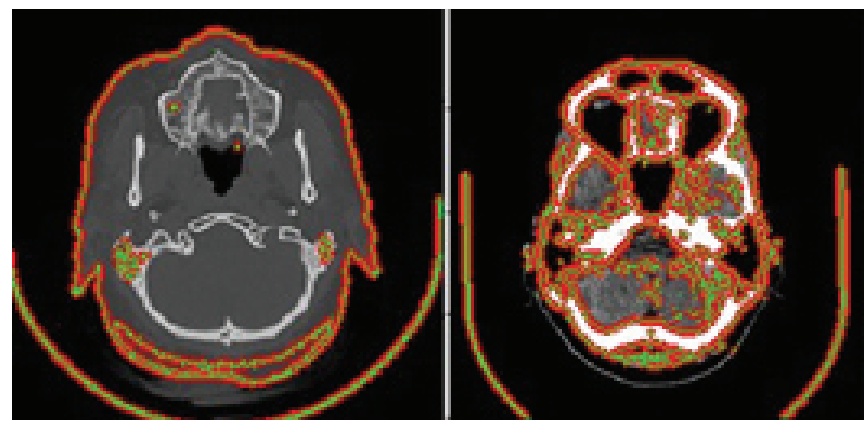

Figure 1. The edges detected using proposed method.
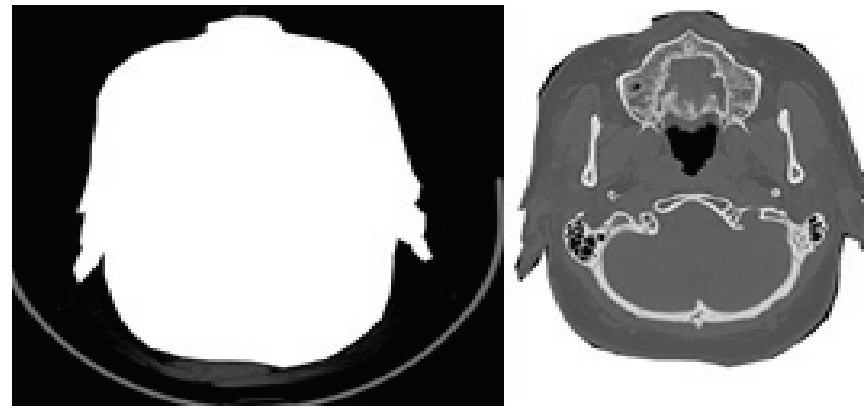

Figure 2. Extracted ROI and Non ROI part of the image.

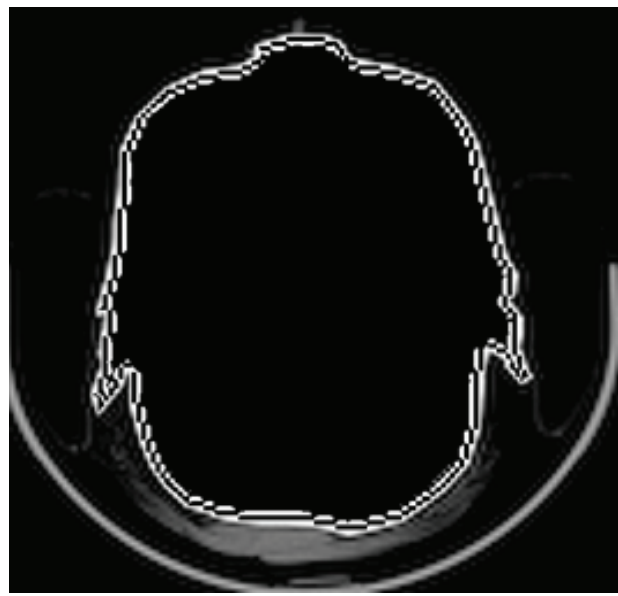

Figure 3. Highly compressed Non Region of interest.

Table 1. Bits per pixel for various images

\begin{tabular}{lccc}
\hline \multicolumn{1}{c}{ SPIHT } & \multicolumn{2}{c}{ EZW } \\
\hline Bits per pixel & PSNR & Bits per pixel & PSNR \\
\hline 2.628 & 40.6536 & 2.9642 & 41.4124 \\
2.614 & 39.973 & 2.8765 & 40.1032 \\
2.623 & 40.7972 & 2.8124 & 40.2122 \\
2.654 & 40.6559 & 2.8376 & 39.7982 \\
2.617 & 40.5055 & 3.0112 & 39.9965 \\
\hline
\end{tabular}

\section{Conclusion}

To overcome the limitations of lossy compression, ROI of the image is compressed using different techniques. It was proposed to segment ROI using Active Contour and apply Biorthogonal wavelet and Embedded ZeroTree (EZW) for compressing the image. Experimental results obtained from the proposed methods were encouraging. After testing the algorithm on different image it was seen that the Peak Signal to Noise Ratio (PSNR) is consistently in the region of 40 which is very feasible.

\section{References}

1. Van Assche S, De Rycke D et al. (2000). Exploitinginterframe redundancies in the lossless compression of $3 \mathrm{D}$ medicalimages, Data Compression Conference, 575.

2. Bullmore E , Fadili J et al. (2004). Wavelets and Functional Magnetic Resonance Imaging of the Human Brain, Neuroimage, vol 23(1), 234-249.

3. Doukas C N, Maglogiannis I et al. (2005). Medical image compression using wavelet transform on mobile devices with ROI coding support, 27th Annual International Conference of the Engineering in Medicine and Biology Society, 2005. IEEE-EMBS 2005, 3779-3784.

4. Dilmaghani R, Ahmadian A et al.,(2003). Multi rate/resolution control in progressive medical image transmission for the region of interest (ROI) using EZW, Proceedings of the 25th Annual International Conference of the IEEE Engineering in Medicine and Biology Society, 2003, vol 1, 818-820.

5. Said and Pearlman W A (1996) A new, fast and efficient image codec based on set partitioning in hierarchical trees, IEEE Transactions on Circuits and Systems for Video Technology, vol 6(3), 243-250.

6. Islam and Pearlman W A (1999) An embedded and efficient low-complexity hierarchical image coder, in Proceeding SPIE, Visual Communications and Image Processing '99, vol 3653, 294-305.

7. Tamilarasi M and Palanisamy V (2009). Fuzzy Based Image Compression on ROI using Optimized Directional Contourlet Transform, International Journal of Recent Trends in Engineering, vol 2(5).

8. Jilani S and Sattar S A (2010). A Fuzzy Neural Networks based EZW Image Compression System, International Journal of Computer Applications (IJCA), vol 2(9), 1-7.

9. Christophe E et al., (2006). Hyperspectral image compression: adapting SPIHT and EZW to anisotopic 3D wavelet coding, IEEE Transactions on Image processing, 1-13.

10. Shingate V S and T.R.Sontakke T R (2010). Still Image Compression Using Embedded Zero tree Wavelet 
Encoding, International Journal of Computer Applications (0975 - 8887), vol 1(7).

11. Babu D V and Alamelu D N (2009). Wavelet Based Medical Image Compression Using ROI EZW, International Journal of Recent Trends in Engineering, vol 1(3), 97-100.

12. Rout S (2003). Orthogonal vs. biorthogonal wavelets for image compression (Doctoral dissertation, Virginia Tech).

13. Kass M, Witkin A et al. (1988). Snakes: Active contour models, International Journal of Computer Vision, vol 1(4), 321-331.

14. Kichenassamy S, Kumar A et al. (1995). Gradient flows and geometric active contour models, Proceedings, Fifth International Conference on Computer Vision, 810-815.
15. Paragios N and Deriche R (1999). Unifying boundary and region-based information for geodesic active tracking, IEEE Computer Society Conference on Computer Vision and Pattern Recognition, vol 2.

16. Kichenassamy S, Kumar A et al. (1995). Gradient flows and geometric active contour models, in Proc. Int. Conf. Computer Vision, 810-815.

17. Goldberg M A, Pivovarov $M$ et al. (1994). Application of wavelet compression to digitized radiographs, American Journal of Roentgenoly, vol 163(2), 463-468.

18. Shapiro J M (1993). Embedded image coding using zerotrees of wavelet coefficients, IEEE Transactions on Signal Processing, vol 41(12), 3445-3462. 